\title{
EVALUACIÓN DEL ESTADO NUTRICIONAL DE GESTANTES EN LA PROVINCIA DE TACNA
}

Guillermo Bornaz Acosta ${ }^{\prime}$ Soledad Bornaz Acosta ${ }^{2}$

RESUMEN

Se evaluó el estado nutricional de 90 pacientes del I, I/ y tercer trimestre de gestación, basado en un estudio de laboratorio consistente en la determinación de los niveles de hematocrito, hemoglobina, proteinas totales y albumina en muestras de sangre. Los resultados fueron comparados con los valores recomendados por el Comite Departamental sobre Nutrición para la Defensa Nacional y por Guzman Barrón A.

Estas pruebas de laboratorio nos indicaron que aproximadamente más del $10 \%$ de embarazadas del I trimestre probablemente padecieron de algún tipo de anemia y más de un $20 \%$ de desnutrición calórico-proteica, estados que se exacerban hasta superar el $40 \%$, conforme avanza el estado de gestación.

\section{A B S TRACT}

The nutritional condition of 90 patients in the first, second and third 3 months of pregnancy was evaluated, based on a laboratory study consisting of the determination of levels of haematocrito, haemoglobin, total proteins and albumen in respective blood samples and albumen in respective blood samples. The results were compared with the values recommended by the DEPARTMENTAL COMMITTEE ON NUTRITION FOR NATIONAL DEFENCE AND GUZMAN BARRON A.

These laboratory samples indicated that approximately more than $10 \%$ of pregnant women in the first three months were probably suffering from some form of anaemia and more than $20 \%$ from protean calorific denutrition, figures which increase, reaching more than $40 \%$, according to the advance of the pregnancy. 


\section{INTRODUCCIÓN}

Se dispone abundante información sobre que al inicio del, embarazo y durante la gestación, una nutrición deficiente afecta el curso del embarazo y el trabajo de parto. De ahí que una buena atención prenatal debe incluir la evaluación del estado nutricional de la embarazada.

Es de origen reciente que el estado nutricional de la embarazada influye profundamente en su salud y la de su hijo (JACOBSON H.N., 1977).

Al evaluar el estado nutricional, es importante identificar los factores que lo colocan en la categoría de mayor riesgo nutricional y decidir qué pacientes necesitarán una valoración especial y una atención en este campo. Al hacer la historia clínica de una paciente embarazada, conviene tener presente si corre riesgo nutricional, en base a parámetros físicos y bioquímicos (HALPPEN S.L., 1990).

Las manifestaciones físicas de una desnutrición (nutrición deficiente), por lo general aparecen tardíamente $\mathrm{y}$, en caso de estar presentes, son inespecíficas y poco evidentes. Los signos físicos de la carencia pueden estar enmascarados por las alteraciones que caracterizan al embarazo normal (TAYLOR K.B., 1983).

Las pruebas específicas de laboratorio son valiosas, pues revelan una nutrición deficiente antes que aparezcan los síntomas clínicos, siendo relativamente específicas.

Es bueno recordar que la información obtenida en el laboratorio, se debe interpretar en el contexto de los ajustes fisiológicos del embarazo que incluyen asimismo los procesos metabólicos. Todas estas adaptaciones pueden alterar los valores de las pruebas hematológicas y bioquímicas, y pueden parecer normales, al compararlos con los que se obtienen en mujeres sanas no embarazadas (HALPERN S.L., 1990).

Los indices de HEMATOCRITOS, HEMOGLOBINA y glóbulos rojos ofrecen diagnósticos de deficiencia de hierro o bien de anemia megaloblástica (deficiencia de folato y cobalamina).

La determinación de proteínas totales y seroalbúmina son indicadores de una desnutrición calórico - proteica; basta tener valores menores de 3.5 gramos de seroalbumina por cada $100 \mathrm{ml}$ de suero o plasma para sugerir una desnutrición energético-proteica.

Las anemias por estados carenciales, que se descubren con mayor frecuencia, se deben a la falta de hierro, folacina y cobalamina, en forma independiente o en varias combinaciones. Durante el embarazo suelen generarse anemias por deficiencia de hierro y folacina.
La anemia es la complicación hematológica más común durante el embarazo y está asociada al incremento de las tasas de nacimientos prematuros, de bajo peso y mortalidad perinatal. La deficiencia de hierro es la causa más común de las anemias. La deficiencia de folacina es la causa más común de anemia megaloblástica durante el embarazo, que puede estar asociada a alteraciones neurológica y otras complicaciones (LOPS V.R., HANTER L.B. y DIVON L.R.; 1994).

Las consecuencias adversas por deficiencia de proteínas durante el embarazo son difíciles de separar de los efectos de deficiencia de calorías en situaciones reales de la vida. Casi todos los casos de proteína limitada están acompañados por limitación de proteínas aprovechables. Bajo ambas circunstancias se han reportado neonatos de menor peso y una mayor incidencia de pre-eclampsia.

Tacna se caracteriza por ser una ciudad de frontera con un costo de vida, quizá el más alto de nuestro país, situación que debe influir sobre el estado nutricional de su población, determinando estados de desnutrición calórico-proteica, especialmente en madres gestantes y niños, por su alta sensibilidad a la carencia de nutrientes.

En este trabajo se pretende realizar un estudio bioquímico de pacientes embarazadas de la ciudad de Tacna, para llevar a cabo la evaluación de su estado nutricional, con la finalidad de darle la atención nutricional y obstétrica adecuada y disminuir el riesgo del embarazo.

\section{MATERIAL Y MÉTODOS}

Se captaron 90 pacientes embarazadas de diferentes trimestres de estado de gestación, a las cuales se les hizo consulta obstétrica, antes de someterlas al estudio bioquímico. De cada paciente se recolectó una muestra de sangre de $5 \mathrm{ml}$. con anticoagulante. Estas pacientes fueron agrupadas de acuerdo a su tiempo de gestación

- Embarazadas del I trimestre: 22

- Embarazadas del II trimestre : 24

- Embarazadas del III trimestre : 44

De las muestras de sangre se tomó una alícuota y se realizaron las siguientes pruebas de laboratorio:

\section{- HEMATOCRITO}

Para determinar el volumen de eritrocitos, expresado en porcentajes de muestras de sangre venosa con anticoagulante, se utilizó el macrométodo de WINTROBE. 


\section{- HEMOGLOBINA}

Para determinar la hemoglobina en sangre expresada en gramos de hemoglobina por $100 \mathrm{ml}$ de sangre venosa, se utilizó el método de la cyanometahemoglobina, que consiste en un método espectrofotométrico basado en la transformación de la hemoglobina en metahemoglobina utilizando ferricianuro de potasio que, al combinarse con el cianuro de potasio, produce cyanometahemoglobina muy estable que puede ser cuantificada, utilizando un espectrofotómetro, usando la longitud de onda de $540 \mathrm{~nm}$.

\section{- PRoteínAs totales}

Para cuantificar las proteínas totales, de la muestra de sangre se tomo una alícuota para aislar el plasma y se determinó las proteínas totales por el método de Biuret, que consiste en la utilización de iones de cobre en medio alcalino y la obtención de un complejo de color azul violeta con las proteínas. Complejo que es cuantificado, utilizando un espectrofotómetro con una longitud de onda de $540 \mathrm{~nm}$.

\section{- ALBÚMINAS}

Para la determinación de albúmina en plasma se utilizó el método de la bromocresolsulfonftaleina que consiste en que la albúmina reacciona específicamente sin separación previa con la forma aniónica de la bromocresolsulfonftaleina (BCF), en presencia de un exceso de colorante en medio taponado a pH 3.8. El aumento de absorbancia a $625 \mathrm{~nm}$. es proporcional a la cantidad de albúmina. Las concentraciones de albúmina en las muestras de plasma, se obtiene multiplicando la absorbancia de la muestra con el factor de calibración, dando un resultado de albúmina en $\mathrm{g} / 100 \mathrm{ml}$ de plasma.

\section{III.RESULTADOS}

Los resultados obtenidos de hemoglobina, expresados en $\mathrm{g} / 100 \mathrm{ml}$. de sangre, se muestran en la Tabla $N^{\circ} 1$ y Figura $N^{\circ} 1$, que fueron tabuladas y comparadas con los valores recomendados por el Comité Interdepartamental sobre la Nutrición para la defensa Nacional, y por Guzmán Barrón, A.
TABLA $N^{\circ} 1$

HEMOGLOBINA EN PACIENTES EMBARAZADAS, EXPRESADAS EN GRAMOS POR $100 \mathrm{ML} \mathrm{DE}$ SANGRE.

\begin{tabular}{|c|c|c|c|c|}
\hline \multirow[b]{2}{*}{ Timestre } & \multicolumn{4}{|c|}{ Hemoglobina $(g / 100 \mathrm{ml})$} \\
\hline & Deficiente & Bajo & Aceptable & Alto \\
\hline 1 & $\begin{array}{l}<10 \\
0 \quad 0.0 \%\end{array}$ & $\begin{array}{l}10-10.9 \\
3-16.9 \%\end{array}$ & $\begin{array}{l}11-14.4 \\
19-86.4 \%\end{array}$ & $\begin{array}{l}>14.5 \\
00.0 \%\end{array}$ \\
\hline II & $\begin{array}{l}<9.5 \\
0 \quad 0.0 \%\end{array}$ & $\begin{array}{l}9.5-10.5 \\
5-20.8 \%\end{array}$ & $\begin{array}{l}10.5-12.9 \\
19-79.2 \%\end{array}$ & $\begin{array}{l}>13 \\
0 \quad 0.0 \%\end{array}$ \\
\hline III & $\begin{array}{l}<9.0 \\
9-20.5 \%\end{array}$ & $\begin{array}{l}9.0-10.4 \\
21-47.7 \%\end{array}$ & $\begin{array}{l}10.5-12.9 \\
14-32.8 \%\end{array}$ & $\begin{array}{l}>13 \\
0 \\
0.0 \%\end{array}$ \\
\hline
\end{tabular}

En las 22 pacientes embarazadas durante el semestre, se encontraron 3 pacientes con niveles bajos de hemoglobina (10 a $10.9 \mathrm{~g} / 100 \mathrm{ml}$ ) que representan el $13.6 \%$; y 19 pacientes con niveles aceptables de hemoglobina (11 a $14.4 \mathrm{~g} / 100 \mathrm{ml}$ ) que representan el $86.4 \%$ del total de embarazadas.

De las 24 pacientes embarazadas del II semestre, se encontraron 5 pacientes con niveles bajos de hemoglobina ( 9.5 a $10.5 \mathrm{~g} / 100 \mathrm{ml}$ ) que representan el $20.8 \%$; y 19 pacientes con niveles de hemoglobina aceptables (10.5 a $12.9 \mathrm{~g} / 100 \mathrm{ml}$ ) que representan el $79.2 \%$ del total de embarazadas.

TABLA $N^{\circ} 2$.

HEMATOCRITO EN PACIENTES EMBARAZADAS EXPRESADO EN PORCENTAJE.

\begin{tabular}{|l|l|l|l|l|l|l|}
\hline \multicolumn{7}{|c|}{ HEMATOCRITO (\%) } \\
\hline Tri. & $24-26$ & $27-29$ & $30-32$ & $33-35$ & $36-38$ & $39-41$ \\
\hline I & 00 & 00 & $29.1 \%$ & $418.2 \%$ & $1045.4 \%$ & $627.3 \%$ \\
\hline II & 00 & 00 & $729.2 \%$ & $833.3 \%$ & $937.5 \%$ & 00 \\
\hline III & $511.4 \%$ & $715.9 \%$ & $1840.9 \%$ & $920.4 \%$ & $511.4 \%$ & 00 \\
\hline
\end{tabular}

Los valores de hematocrito determinados en muestras de sangre de las pacientes embarazadas, se encuentran en la TABLA 2 y Figura 2, donde ningún paciente del I y II trimestre presentaron hematocrito del 24 a $26 \%$, pero sí presentaron 5 pacientes del III 
trimestre. Igualmente, ningún paciente del I y II trimestre presentaron hematocritos del 27 al $29 \%$, pero sí 7 del III trimestre. Hematocritos del 29 al $32 \%$ fueron presentados por 2, 7 y 18 pacientes del I, II y III trimestre respectivamente. 4, 8 y 9 pacientes del I, II y III trimestre respectivamente, presentaron hematocritos del 33 al $35 \%$. Hematrocritos del 36 al $38 \%$ fueron presentados por 10, 9 y 5 pacientes del I, II y III trimestre, respectivamente. Solamente 7 pacientes del I semestre presentaron hematocritos del 39 al $41 \%$.

Los resultados de la determinación de proteínas totales en muestras de plasma de las pacientes embarazadas, se encuentran en la tabla №3 y en la Figura № 3 . De las 22 pacientes grávidas del I semestre, 2 de ellas presentaron niveles deficientes de proteínas séricas totales (menor de $6.0 \mathrm{~g} / 100 \mathrm{ml}$ de suero o plasma); 3 pacientes con niveles bajos ( 6 a $6.4 \mathrm{~g} / 100$ $\mathrm{ml}$ de suero o plasma); y 17 pacientes con niveles aceptables (6.5 a $6.9 \mathrm{~g} / 100 \mathrm{ml}$ de suero o sangre). Ninguna paciente presentó niveles altos de proteínas totales (mayor que $7 \mathrm{~g} / 100 \mathrm{ml}$ de suero o plasma).

TABLA N $N^{\circ}$.

PROTEÍNAS SÉRICAS TOTALES EN PACIENTES EMBARAZADAS, EXPRESADAS EN GRAMOS POR CADA 100 ML DE SUERO O PLASMA.

\begin{tabular}{|c|c|c|c|c|}
\hline \multirow{2}{*}{ Trimestre } & \multicolumn{4}{|c|}{ Proteínas Séricas Totales } \\
\cline { 2 - 5 } & Deficiente & \multicolumn{1}{|c|}{ Bajo } & Aceptable & Alto \\
\hline I & $<6.0$ & $\begin{array}{l}6.0-6.4 \\
0313.6 \%\end{array}$ & $\begin{array}{l}6.5-6.9 \\
17\end{array}$ & $>77.3 \%$ \\
& $029.1 \%$ & 00 \\
\hline II & $<5.5$ & $\begin{array}{l}5.5-5.9 \\
07\end{array}$ & $\begin{array}{l}6.0-6.9 \\
16\end{array}$ & $>7.0$ \\
& $014.2 \%$ & 00 \\
\hline III & $<5.5$ & $\begin{array}{l}5.5-5.9 \\
2045.5 \%\end{array}$ & $\begin{array}{l}6.0-6.9 \\
1125.0 \%\end{array}$ & $>7.0$ \\
\hline
\end{tabular}

De las 24 pacientes del II trimestre, una presentó niveles deficientes de proteínas séricas totales (menor que $5.5 \mathrm{~g} / 100 \mathrm{ml}$ de suero o plasma); 7 presentaron niveles bajos ( 5.5 a $5.9 \mathrm{~g} / 100 \mathrm{ml}$ de suero o plasma); $y$ 16 pacientes con niveles aceptables $(6.5$ a $6.9 \mathrm{~g} / 100$ $\mathrm{ml}$ de suero o plasma). Ninguna paciente presentó niveles altos (mayor de $7 \mathrm{~g} / 100 \mathrm{ml}$ de suero o plasma).

Del tercer trimestre, 13 pacientes presentaron niveles deficientes de proteínas séricas totales (menor de 5.5 $\mathrm{g} / 100 \mathrm{ml}$ de suero o plasma); 20 presentaron niveles bajos (5.5 a $5.9 \mathrm{~g} / 100 \mathrm{ml}$ de suero o plasma); y 11 , niveles aceptables (6 a $6.9 \mathrm{~g} / 100 \mathrm{ml}$ de suero o plasma). En ningún caso se presentaron niveles altos (mayor de $7 \mathrm{~g} / 100 \mathrm{ml}$ de suero o plasma).

Los resultados de las determinaciones de albúmina sérica se muestran en la tabla $\mathrm{N}^{\circ} 4$ y Figura $\mathrm{N}^{\circ} 4$. De las 22 pacientes del primer semestre, 2 presentaron niveles deficientes de albúmina sérica ( menor de 3.3 $\mathrm{g} / 100 \mathrm{ml}$ de suero o plasma), 3 con niveles bajos (3.3 a $3.9 \mathrm{~g} / 100 \mathrm{ml}$ de suero o plasma) y 17 con niveles aceptables ( 4.0 a $4.9 \mathrm{~g} / 100 \mathrm{ml}$ de suero o plasma). Ningún paciente mostró niveles altos de albúmina sérica (mayor de $5 \mathrm{~g} / 100 \mathrm{ml}$ de suero o plasma).

TABLA $N^{\circ} 4$.

ALBÚMINA SÉRICA EN PACIENTES

EMBARAZADAS, EXPRESADA EN GRAMOS POR

CADA $100 \mathrm{ml}$ DE SUERO O PLASMA.

\begin{tabular}{|c|c|c|c|c|}
\hline \multirow[b]{2}{*}{ Trim. } & \multicolumn{4}{|c|}{ Albúmina Sérica } \\
\hline & Deficiente & Bajo & Aceptable & Alto \\
\hline 1 & $\begin{array}{l}<3.3 \\
02 \quad 9.1 \%\end{array}$ & 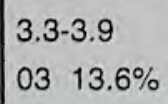 & $\begin{array}{l}4.0-4.9 \\
17 \quad 77.3 \%\end{array}$ & $\begin{array}{c}>5.0 \\
00\end{array}$ \\
\hline II & $\begin{array}{l}<3.0 \\
02 \quad 8.3 \%\end{array}$ & $\begin{array}{l}3.0-3.9 \\
07 \quad 29.2 \%\end{array}$ & $\begin{array}{l}4.0-4.9 \\
1562.5 \%\end{array}$ & $\begin{array}{l}>5.0 \\
00\end{array}$ \\
\hline III & $\begin{array}{l}<3.0 \\
1227.3 \%\end{array}$ & 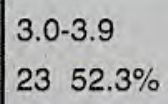 & $\begin{array}{l}4.0-4.9 \\
0920.4 \%\end{array}$ & $\begin{array}{l}>5.0 \\
00\end{array}$ \\
\hline
\end{tabular}

De las 24 pacientes del II trimestre, 2 presentaron niveles deficientes de albúmina sérica, 7 con niveles bajos y 15 con niveles aceptables. En ningún caso se presentaron niveles altos de albúmina plasmática.

Doce pacientes del III trimestre presentaron niveles deficientes de albúmina sérica, 23 con niveles bajos y 9 con niveles aceptables. Ninguna paciente mostró niveles elevados de albúmina sérica.

\section{IV.DISCUSIÓN}

Debido a que se ha comprobado que tanto al inicio como durante el embarazo, una nutrición deficiente afecta el resultado de la gestación, una atención prenatal detallada ayuda a evaluar el estado nutricional de la madre. No es raro encontrar en países en vías de desarrollo, deficiencias nutritivas claras, pero también se suelen presentar en madres aparentemente sanas, 
estados de desnutrición subclínica o latente. Al realizar la evaluación de la mujer embarazada, no se busca únicamente reconocer las fallas nutritivas, sino también los factores que determinan un embarazo con riesgo y establecer si la paciente necesitará pruebas y cuidados especiales. Por tales razones es necesario recordar las categorías de riesgo nutricional durante el embarazo (SEYMOUR L.H., 1990).

En los resultados de hematocrito se observa claramente que el $9.1 \%, 29.2 \%$ y el $67.9 \%$ de las embarazadas del 1, II y III trimestre, respectivamente, presentaron volúmenes globulares (hematocrito) inferiores al $33 \%$, lo que nos indica que son pacientes con riesgo nutricional; además del $69.7 \%$ de las embarazadas del III trimestre, el $27.3 \%$ presentaron hematocritos deficientes (menores al $30 \%$ ), que nos indican un mayor riesgo.

Los niveles de hemoglobina mostrados en RESULTADOS, nos demuestran que conforme avanza el embarazo, el riesgo nutricional se incrementa; de un $13.6 \%$ de pacientes del I trimestre con niveles bajos de hemoglobina, a un $20.8 \%$ de pacientes del II trimestre, hasta un $68.2 \%$ de pacientes del III trimestre con niveles deficientes y bajos.

Los valores de hematocrito y hemoglobina en las pacientes embarazadas, presentan una correlación a través del embarazo, indicándonos que el riesgo se incrementa conforme avanza el embarazo. Estos resultados nos indican que las pacientes con riesgo, probablemente estén sufriendo de algún tipo de anemia.

Durante el embarazo se suele asociar una mayor actividad hematológica. Aumentan el volumen del plasma y la masa de globulos rojos. Sin embargo, esto no ocurre en la misma proporción, por lo que la mayor elevación del primero ocasiona una disminución gradual en el hematocrito que alcanza su nivel más bajo a la $32^{\circ}$ semana de gestación (OLSON C.M., 1994). A medida que la masa de glóbulos rojos aumenta, hacia fines del embarazo, el hematocrito llega de nuevo a los valores existentes antes de que éste se iniciara. Después del parto, el hematocrito tiende a elevarse como resultado de la diuresis fisiológica y contracción del volumen sanguíneo (OLSON C.M.,1994).

El National Research Council recomienda más de $18 \mathrm{mg}$ al día de de fierro para mujeres que estén o no embarazadas. Ni siquiera la mejor dieta puede aportar esta cantidad, por lo que es necesario suministrar hierro complementario para que el cuidado prenatal sea adecuado. Si éste no se administra, aunque la mujer tenga un nivel excelente de nutrición durante el embarazo, al final terminará con un déficit en sus depositos de fierro; por el contrario, si la alimentación prenatal ha sido insuficiente, desarrollará anemia (THANANGKULL O. 1994).
Las anemias por estados carenciales, que se descubren con mayor frecuencia en la práctica clínica, se deben a la falta de fierro, folacina y cobalamina, en forma independiente o en varias combinaciones. Durante el embarazo se suelen generar anemias por deficiencia de fierro y folacina (BELL W.R., 1987).

La aparición de deficiencia de fierro en el embarazo afecta sucesivamente a los diferentes compartimientos de tejidos. Cuando las pérdidas rebasan la absorción, ocurre primero la reducción de las reservas de fierro en hígado, bazo y médula ósea y luego se produce desaturación de fierro de la transferrina. A veces se dice que disminuye el número de eritrocitos, pero esto no es así. Lo que sucede es que el número creciente se vuelve de tamaño menor que lo normal y por último, merma la concentración de hemoglobina del eritrocito. La anemia ferropénica plena es microcítica hipocrómica. Si bien, no es indicio sensible de depleción de fierro, los microcitos aparecen en fase temprana (OLSON C.M.,1994).

La deficiencia de fierro es una de las formas más comunes de malnutrición a nivel mundial. La baja ingestión de fierro dietético, está asociada a una disminución de su absorción, lo que se agrava con infestación parasitaria que causa pérdida sanguínea crónica de los intestinos o vias urinarias (OLSON C.M.,1994).

El término deficiencia férrica designa un padecimiento en el que se depleta el contenido total de fierro en el organismo. Si es latente, los depósitos de fierro se vacian, pero los niveles de hemoglobina permanecen sobre el límite inferior de lo normal, por lo general este estado se puede detectar por una reducción de hierro en el suero. Si este último es inferior a $42 \mathrm{ug} / 100 \mathrm{ml}$ puede también indicar ausencia de almacenamiento medular en la mayoría de mujeres embarazadas. Las mujeres en este estado muestran un hematocrito del 30 al $33 \%$. La anemia en las mujeres embarazadas se define en base a un hematocrito menor del $33 \%$ y una hemoglobina inferior a $10 \mathrm{~g} / 100 \mathrm{ml}$. El empleo de estos parámetros nos permite detectar una deficiencia latente de fierro (Seymour L.H., 1990).

La anemia es la complicación hematológica más común durante el embarazo y está asociada al incremento de las tasas de nacimientos prematuros de bajo peso y mortalidad perinatal. La deficiencia de fierro es la causa más común de las anemias y las mujeres embarazadas se pueden beneficiar con darles diariamente un suplemento de 32 a $68 \mathrm{mg}$ de Fe. La deficiencia de ácido fólico es la causa más común de anemia megaloblástica durante el embarazo, que está asociada a alteraciones del tubo neural y otras complicaciones. Es recomendable un suplemento diario de $4 \mathrm{mg}$ de ácido fólico (OLSON C.M.,1994). 
Una explicación de cómo las anemias provocan alteraciones durante el embarazo; es que la nutrición puede influenciar en el intercambio de oxígeno a través de la placenta. Cada gramo de hemoglobina transporta $1.34 \mathrm{ml}$ de oxígeno. En concentraciones normales la hemoglobina puede entregar $16 \mathrm{ml}$ de oxígeno por cada $100 \mathrm{ml}$ de sangre a la placenta. Si los niveles de hemoglobina materna son disminuido por deficiencia de fierro, el suministro de oxígeno por cada $100 \mathrm{ml}$ de sangre es reducido. Puesto que el feto puede tolerar una pequeña variación en la tasa de oxígeno suministrado, la madre debe compensar esa variación con un incremento en el rendimiento cardiaco. Caso contrario, se va a tener un suministro disminuido de oxígeno, lo que va a provocar una baja en la combustión de nutrientes y con ello una merma en la producción de energia que va a afectar el crecimiento intrauterino (BELL W.R.,1990).

Para la profilaxis de la deficiencia de fierro se puede suministrar complementos, como sales ferrosas simples por via oral en cantidades de 30 a $60 \mathrm{mg}$ por día, durante el embarazo y los 2 a 3 meses que siguen al parto. Para el tratamiento de deficiencia de fierro se necesitan cantidades mayores, como de 200 a 300 mg de sulfato ferroso (BELL W.R.,1987).

Los resultados de proteínas séricas totales, nos muestran que conforme avanza el embarazo, el porcentaje de pacientes grávidas con niveles deficientes y bajos de proteinas totales se incrementa al término. De $22.7 \%, 33.4 \%$ a $75 \%$ se incrementa en el I, II y II trimestre respectivamente. Lo mismo ocurre con los valores de albúmina sérica presentados en RESULTADOS, donde de un $22.6 \%$ de pacientes con niveles deficientes y bajos de albúmina al inicio del embarazo (primer trimestre), se incrementa a un $79.3 \%$ al final del embarazo (tercer trimestre). Este parámetro nos indica que las pacientes consideradas en dichos porcentajes, presentan un embarazo con riesgo nutricional. Una albúmina menor de $3.5 \mathrm{~g} / 100 \mathrm{ml}$ de suero o plasma es indicativo que la paciente presenta probable desnutrición proteica.

No se conoce con exactitud la cantidad de proteinas que se deposita durante la gestación. A partir de estudios de composición corporal, se ha calculado que en promedio, aproximadamente, 925 gramos de proteínas se depositan en el feto y tejidos accesorios de la madre. Las proteínas pueden almacenarse en los tejidos maternos durante las primeras etapas del embarazo para utilizarse más adelante, cuando aumentan las demandas de crecimiento del feto. La retención de nitrógeno que se observa en la mujer embarazada sana y correctamente alimentada es el doble de lo que se predijo cuando se analizaron los tejidos (OLSON C.M.,1994).

Los informes relativos a los beneficios de un generoso aporte de proteínas para la madre, son sugerentes y sin embargo, discutibles. La información epidemiológica no es convincente, debido a que una ingestión baja de proteínas (que deteriora la reproducción) se acompaña casi siempre de una baja ingestión calórica. Se ha encontrado que la complementación de energía sola o acompañada con proteínas, es benéfica en aquellas mujeres cuyas dietas contienen cantidades pequeñas de éstas. Por lo que se recomienda un suplemento de proteínas de 20 gramos (THANANGKULL, O., 1994).

Para que el crecimiento y desarrollo del feto sean adecuados, es necesario que la madre suministre nutrimentos al feto constantemente, la disponibilidad de éstos dependerá de su concentración en el torrente sanguineo materno, del flujo sanguíneo de la madre que atraviesa la placenta, del paso de los nutrientes a la circulación fetal a través de la placenta y de la suficiente circulación fetal. De ahi que los siguientes factores pueden deteriorar el crecimiento fetal: una dieta insuficiente, absorción inadecuada nivel intestinal, metabolismo anormal en el organismo materno, circulación insuficiente en la placenta, y utilización anormal de nutrientes por el feto.

Una disminución en el aporte nutricional al crecimiento fetal representa el $60 \%$ de los casos de retraso clínico de crecimiento. El defecto fundamental implica el deterioro en el suministro de nutrientes que atraviesan la placenta. Es probable que el común denominador de este tipo de retraso sea la hipoxia fetal, generada por una anemia, que puede estar acompañada por una deficiencia calórico - proteica. Hay interferencia tanto en el desarrollo hiperplásico como hipertrófico. Estos recién nacidos presentarán enflaquecimiento con un aumento de tamaño de la cabeza con relación al del cuerpo (SEYMOUR L.H., 1990).

En casi el $50 \%$ de casos, el retraso de debe a factores que derivan de la madre. Entre ellos está la nutrición deficiente, según se puede advertir por su poco peso antes del embarazo y por el aumento reducido durante el mismo. Existen otros factores, de los cuales, algunos llevan de manera indirecta a la desnutrición materna, como situaciones socioeconómicas, tabaquismo, alcoholismo, adolescencia y mala salud (OLSON C.M.,1994).

Las embarazadas del departamento de Tacna presentan un grado de nutrición inadecuado, antes de la concepción y durante el embarazo, evidenciándose en el tercer trimestre.

La combinación de estos parámetros: hematocrito, hemoglobina, proteínas totales y albúmina sérica; aparecen como indicadores sensibles para detectar embarazos con riesgo y de alto riesgo nutricional. Posteriormente se deberá darles la debida atención prenatal y asesoramiento nutricional, para evitar neonatos prematuros, de bajo peso y menor tasa de mortalidad perinatal. 

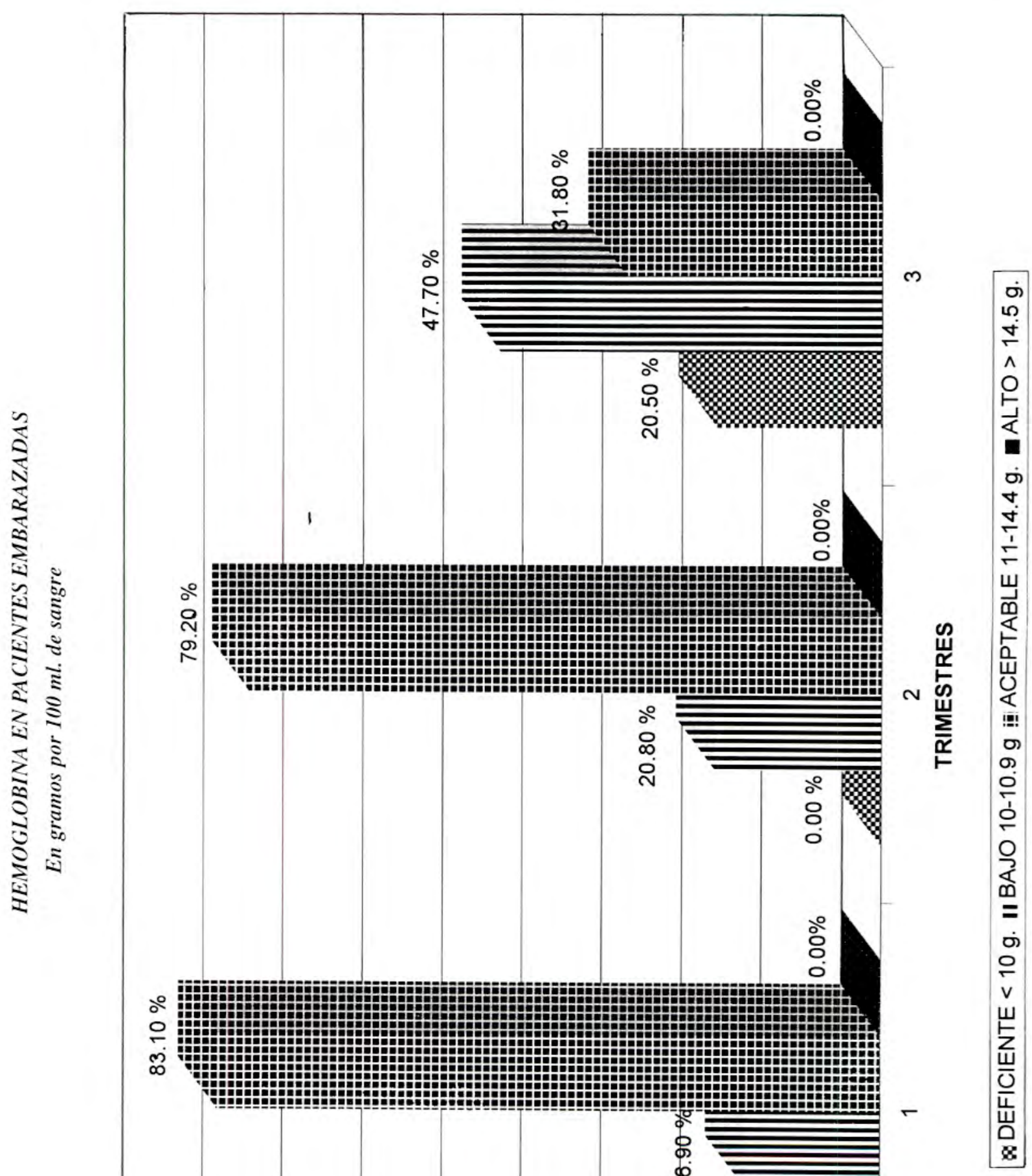







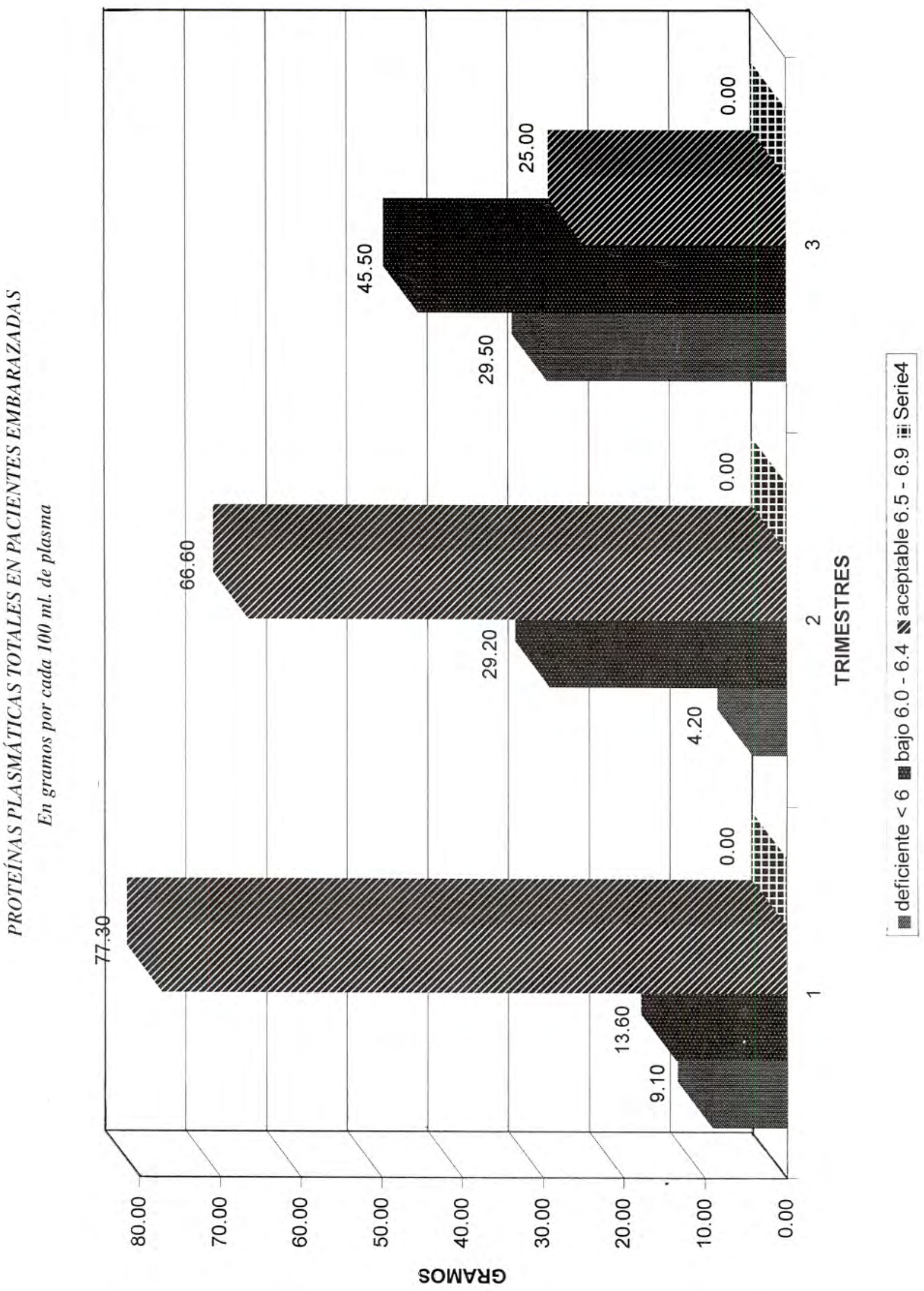




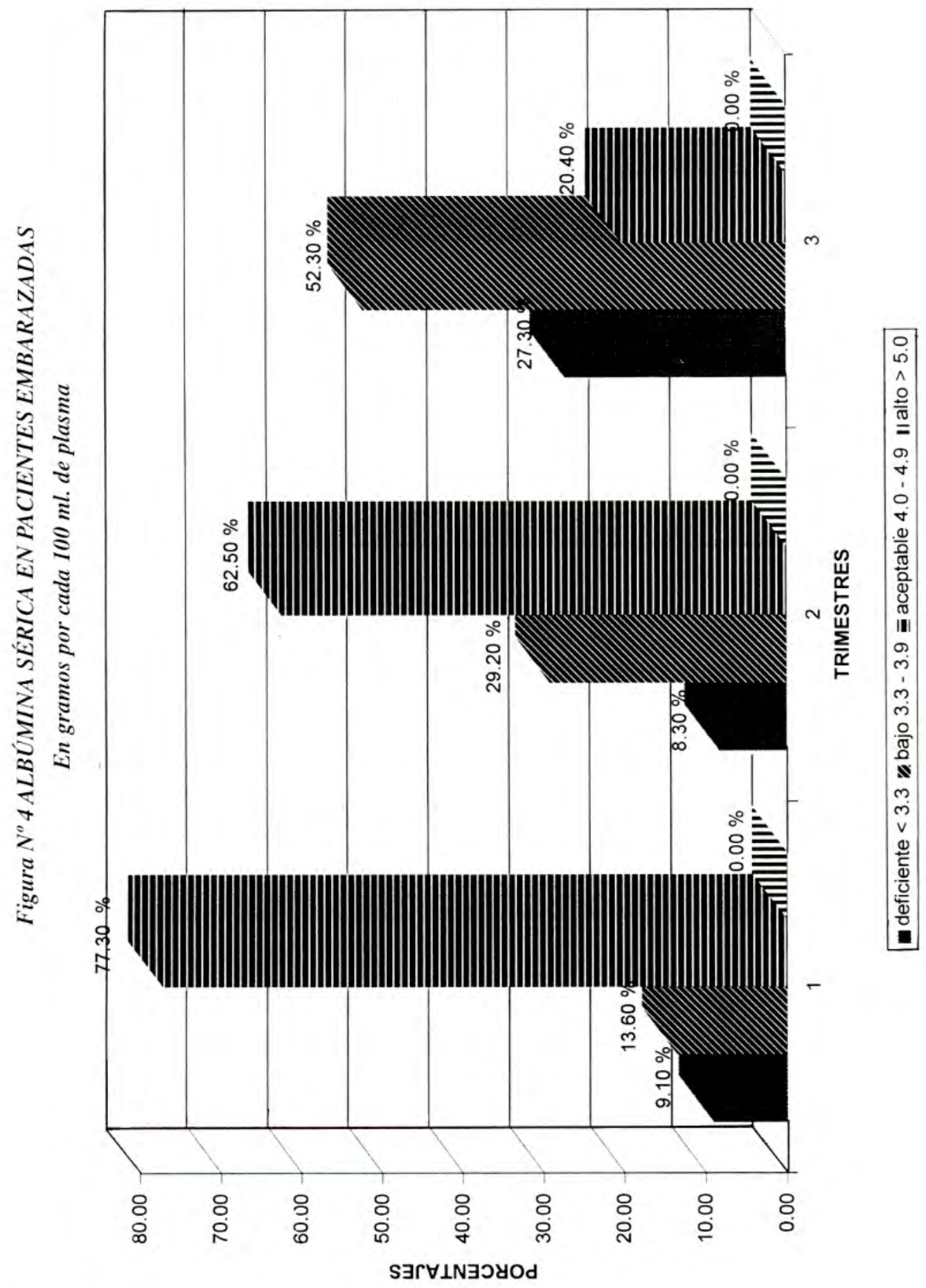

\section{BIBLIOGRAFÍA}

BELL, W.R. Hematologic in pregnancy. Med Clin North Am, 61:165, 1987.

SEYMOUR L.H. Manual de nutrición clínica. Ed. Noriega Limusa, México, 1990.

JACOBSON H.M. Current concepts in nutrition. N Engl, J. Med, 297:1051, 1987.

OLSOM C.M. Promoting positive nutritional practices during pregnancy and lactation. Am J Clin Nutr, febrero, 1994.

THANANGKUL O. y Col. Iron and folate supplementation during pregnancy. Maternal and fetal consequences. Adv. Exp. Med. Biol. 352: 151-6, 1994.

GUZMÁN BARRÓN A. y Col. Nutrición Humana, tomo I, 1980. Hill, 1985. 


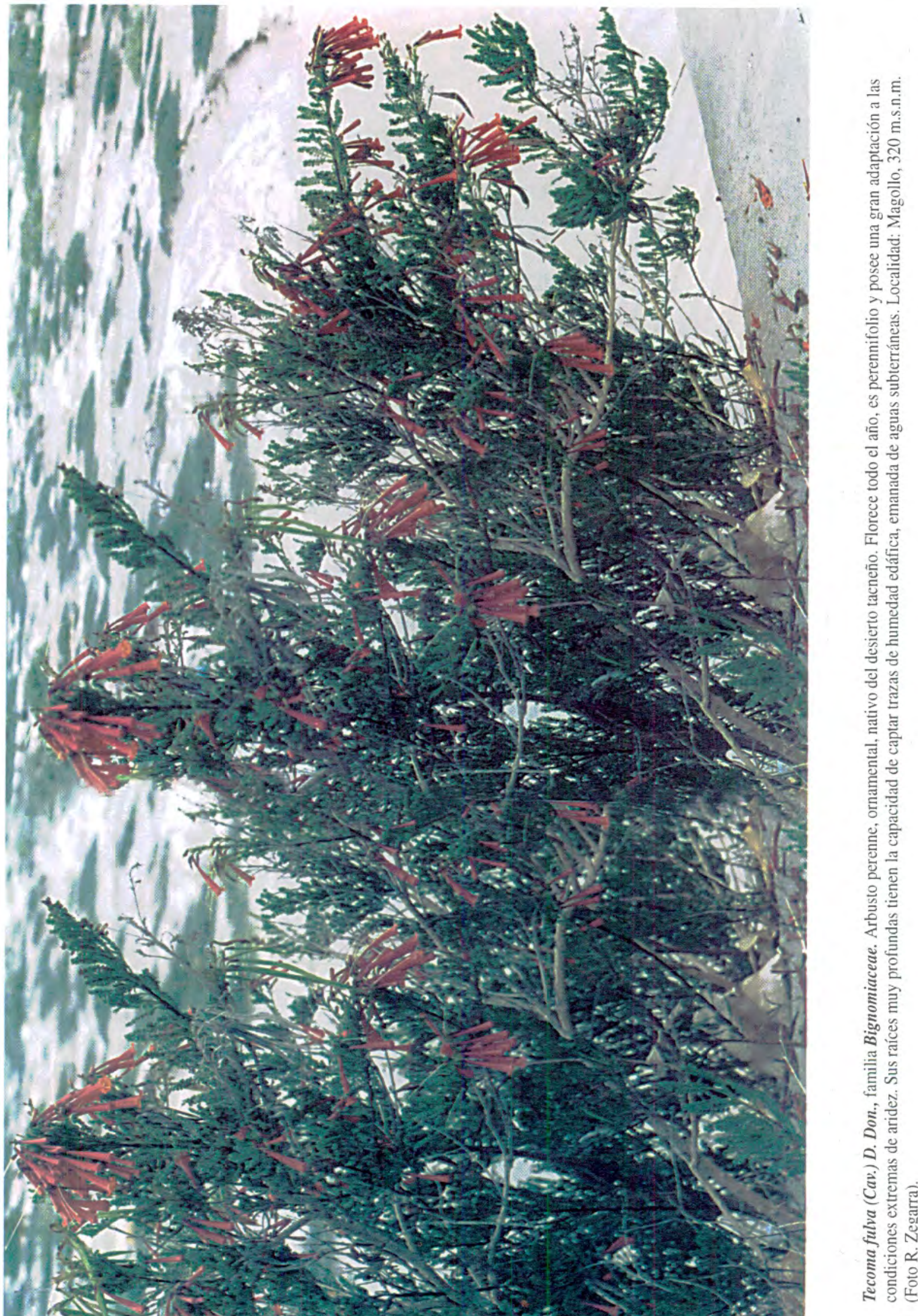

